\title{
A Hierarchy of Classes of Bounded Bitolerance Orders
}

\author{
Garth Isaak* \\ Department of Mathematics \\ Lehigh University \\ Lehigh, PA 18015 \\ Kathryn L. Nyman \\ Department of Mathematics \\ Cornell University \\ Ithaca, NY 14853 \\ Ann N. Trenk ${ }^{\dagger}$ \\ Department of Mathematics \\ Wellesley College \\ Wellesley, MA 02481
}

July 30, 2001

\begin{abstract}
In this paper we extend the work of Bogart and Trenk [3] and Fishburn and Trotter [6] in studying different classes of bitolerance orders. We provide a more comprehensive list of classes of bitolerance orders and prove equality between some of these classes in general and other classes in the bipartite domain. We also provide separating examples between unequal classes of bitolerance orders.
\end{abstract}

\section{Introduction}

An ordered set or poset $P=(V, \prec)$ consists of a set $V$ and a binary relation $\prec$ on $V$ which is irreflexive, transitive and therefore antisymmetric. In this

\footnotetext{
${ }^{*}$ Research supported in part by the Reidler Foundation.

${ }^{\dagger}$ Research supported in part by the American Association of University Women.
} 
paper we consider only finite posets. If $x \prec y$ or $y \prec x$ we say that $x$ and $y$ are comparable; otherwise we say they are incomparable and write $x \| y$. An interval order is a poset $P=(V, \prec)$ that has a representation in which $v \in V$ is assigned an interval $I_{v}$ on the real line so that $x \prec y$ if and only if $I_{x}$ is completely to the left of $I_{y}$.

One application of interval orders arises in the scheduling of events. If $I_{v}$ represents the meeting time period for event $v$ then events $x$ and $y$ do not conflict (and can be assigned to the same room) if and only if $x$ and $y$ are comparable. Unfortunately, this strict notion of conflict can lead to difficulties such as there not being enough rooms to meet the demand for meeting space. One solution adds some flexibility by assigning both an interval $I_{v}=[L(v), R(v)]$ and a real tolerance, $t_{v}$ where $0<t_{v} \leq\left|I_{v}\right|$ to each event. In this interpretation, $x \prec y$ if and only if $L(x)<L(y)$ and $\left|I_{x} \cap I_{y}\right|<\min \left\{t_{x}, t_{y}\right\}$. Thus two events may still be comparable even if their assigned intervals intersect. We can think of the intersection as a time period in which they would share the resource, such as a meeting room.

Posets that can be represented in this way are called bounded tolerance orders. They were first studied in [8] and [9] via their incomparability graphs as the class of bounded tolerance graphs.

In a representation of a bounded tolerance order, if $I_{x} \subseteq I_{y}$ then $\mid I_{x} \cap$ $I_{y}|=| I_{x} \mid \geq t_{x} \geq \min \left\{t_{x}, t_{y}\right\}$ so $x \| y$. Thus we can have a non-empty intersection between the intervals assigned to comparable elements only when that intersection is at the beginning or the end of each interval. An even broader class of orders was introduced in [3] motivated by the idea that the length of intersection a meeting may be willing to tolerate at the beginning could be different from the amount it would tolerate at the end.

Definition 1. An order $P=(V, \prec)$ is a bounded bitolerance order if each $v \in V$ can be assigned a real interval $I_{v}=[L(v), R(v)]$ and two additional tolerant points $p(v), q(v) \in I_{v}$ satisfying $p(v) \neq L(v)$ and $q(v) \neq R(v)$ so that $x \prec y$ if and only if $R(x)<p(y)$ and $q(x)<L(y)$. The quantity $t_{l}(v)=p(v)-L(v)>0$ is the left tolerance of $v$ and $t_{r}(v)=R(v)-q(v)>0$ is the right tolerance of $v$. The collection $\langle\mathcal{I}, p, q\rangle$, where $\mathcal{I}=\left\{I_{v} \mid v \in V\right\}$, $p=\{p(v) \mid v \in V\}$ and $q=\{q(v) \mid v \in V\}$ is called a bounded bitolerance representation.

Figure 1 shows the order $\mathbf{3}+\mathbf{2}$ and a bounded bitolerance representation of it. In this and later representations, we use rectangles (resp. ellipses) to denote the location of tolerant points $p(v)$ (resp. $q(v)$ ). More generally, the order $\mathbf{r}+\mathbf{s}$ consists of two chains: $x_{1} \prec x_{2} \prec \cdots \prec x_{r}$ and $y_{1} \prec y_{2} \prec$ $\cdots \prec y_{s}$, with $x_{i}$ incomparable to $y_{j}$ for every $i$ and $j$. Orders of the form $\mathbf{r}+\mathbf{s}$ appear repeatedly in Section 2 .

The class of bounded bitolerance orders is equivalent to two well-known classes of posets. The first are posets of interval dimension at most two 


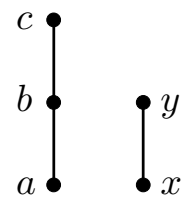

$3+2$
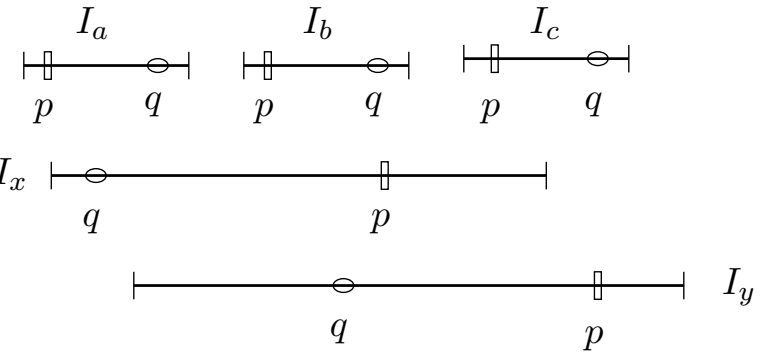

Figure 1: The order $\mathbf{3}+\mathbf{2}$ and a bounded bitolerance representation of it.

(denoted by $i d i m \leq 2$ ), that is, posets that can be written as the intersection of two interval orders. The second are trapezoid orders, introduced in [4]. Dagan et al. [4] prove that these two classes are equivalent, and Langley [11] observed that they are also equivalent to the class of bounded bitolerance orders.

Subclasses of bounded bitolerance orders have been studied based on three kinds of restrictions.

\section{Restrictions on Intervals $I_{v}$}

Definition 2. (Unit): $P$ is a unit bitolerance order if it has a bounded bitolerance representation $\langle\mathcal{I}, p, q\rangle$ in which $\left|I_{x}\right|=\left|I_{y}\right|$ for all $x, y \in V$.

Definition 3. (Proper): $P$ is a proper bitolerance order if it has a bounded bitolerance representation $\langle\mathcal{I}, p, q\rangle$ in which $I_{x} \not \subset I_{y}$ for all $x, y \in V$.

\section{Restrictions on Tolerant Points $p(v), q(v)$}

Definition 4. (Point-core): $P$ is a point-core bitolerance order (or pointcore order) if it has a bounded bitolerance representation $\langle\mathcal{I}, p, q\rangle$ in which $p(v)=q(v)$ for all $v \in V$. In this case we call this point the splitting point of $I_{v}$, denote it by $f(v)=p(v)=q(v)$, and denote the representation by $\langle\mathcal{I}, f\rangle$.

Definition 5. (Totally Bounded): $P$ is a totally bounded bitolerance order if it has a bounded bitolerance representation $\langle\mathcal{I}, p, q\rangle$ in which $p(v) \leq$ $q(v)$ for all $v \in V$.

\section{Restrictions on Left and Right Tolerance}

Definition 6. (Tolerance): $P$ is a bounded tolerance order if it has a bounded bitolerance representation $\langle\mathcal{I}, p, q\rangle$ in which $t_{l}(v)=t_{r}(v)$ for all $v \in V$. In this case we write $t_{v}=t_{l}(v)=t_{r}(v)$. 


\begin{tabular}{|lll|}
\hline interval length & $p$ and $q$ & $t_{l}$ and $t_{r}$ \\
\hline 1. unit & a. point-core & i. tolerance \\
2. proper & b. totally bounded & ii. bitolerance \\
3. - & c. - & \\
\hline
\end{tabular}

Figure 2: Restrictions which can be placed on bounded bitolerance representations.

Analogous to the totally bounded restriction is one in which $q(v) \leq p(v)$ for all $v$. However, this does not place a substantive restriction on bounded bitolerance representations, as we show in Proposition 8. One can also show that the definition of a bounded tolerance order given in Definition 6 agrees with the one presented earlier.

Each of the three categories of restriction is independent. For example, a restriction on interval lengths (such as "unit") can be applied with or without a restriction on $p$ and $q$ (such as "totally bounded"). In addition, we also have the option of not applying any of the restrictions in a category. The chart in Figure 2 summarizes the possible restrictions, where "_" denotes no restriction in that category.

Choosing one restriction from each category gives a total of 18 classes of bounded bitolerance orders, some of which turn out to be equivalent. These classes are listed by their abbreviations in Figure 3, and referred to by these abbreviations throughout this paper. For example, (1bii) is the class of unit totally bounded bitolerance orders, while (2ci) is the class of proper tolerance orders.

Bogart and Trenk [3] consider nine of the classes (in the order listed below) which arise from allowing one restriction from the first two categories and one restriction from the third: (1ci), (3ai), (1cii), (2ci), (2cii), (3bi), (3bii), (3ci), and (3cii). Fishburn and Trotter [6] consider some of these classes plus point-core bitolerance orders (3aii) which they call split interval orders and unit point-core bitolerance orders (1aii), which they call split semiorders.

We end this section with a lemma about distinctness of endpoints and tolerant points and a note afterwards about distinctness of tolerances. In many previous papers on tolerance graphs and orders it is noted without proof that one can find a representation with distinct endpoints, tolerant points and tolerances. This fact is not immediately obvious when additional conditions such as unit or proper are assumed. Indeed in [6] there is a lengthy proof of two variants on this comment. Here we provide a short proof.

Lemma 7. If $P=(V, \prec)$ is a member of any of the 18 classes of bitol- 
erance orders obtained using the restrictions in Figure 2, then it has a representation in which all endpoints and tolerant points are distinct. If $P$ is a unit or proper interval order, then it has a representation in which all endpoints are distinct.

Proof. Fix a bounded bitolerance representation $\langle\mathcal{I}, p, q\rangle$ of $P=(V, E)$. Assume that $V=\{1,2, \ldots,|V|\}$ and let $\epsilon$ be the smallest positive distance between two distinct points in $\{L(x), p(x), q(x), R(x) \mid x \in V\}$. Form a new representation with $L^{\prime}(x)=L(x)-\epsilon / 10+\epsilon / 10^{x+2}, p^{\prime}(x)=p(x)-$ $\epsilon / 10^{2}+\epsilon / 10^{x+2}, q^{\prime}(x)=q(x)+\epsilon / 10^{2}+\epsilon / 10^{x+2}$ and $R^{\prime}(x)=R(x)+$ $\epsilon / 10+\epsilon / 10^{x+2}$. The following are straightforward to check from the definitions of $L^{\prime}(x), p^{\prime}(x), q^{\prime}(x), R^{\prime}(x)$ and the use of terms involving $\epsilon$ : the prime representation has all endpoints and tolerant points distinct, if the original representation was proper or unit or totally bounded or satisfied the 'tolerance' property then so is the prime representation. It remains to check that the prime representation also represents $P$. If $p(y)-R(x)>0$ then by the choice of $\epsilon, p(y)-R(x)>\epsilon$ and thus $p^{\prime}(y)-R^{\prime}(x)=(p(y)-$ $\left.\epsilon / 10^{2}+\epsilon / 10^{y+2}\right)-\left(R(x)+\epsilon / 10+\epsilon / 10^{x+2}\right)>0$. If $p(y)-R(x) \leq 0$ then $p^{\prime}(y)-R^{\prime}(x)=\left(p(y)-\epsilon / 10^{2}+\epsilon / 10^{y+2}\right)-\left(R(x)+\epsilon / 10+\epsilon / 10^{x+2}\right) \leq 0$ since $\left(-\epsilon / 10^{2}+\epsilon / 10^{y+2}\right)-\left(\epsilon / 10+\epsilon / 10^{x+2}\right) \leq 0$. So $R(x)<p(y) \Leftrightarrow R^{\prime}(x)<p^{\prime}(y)$. Similarly $q(x)<L(y) \Leftrightarrow q^{\prime}(x)<L^{\prime}(y)$.

For point-core representations do as above but omit the terms $\epsilon / 10^{2}$ from $p^{\prime}(x)$ and $q^{\prime}(x)$ so that $p(x)=q(x) \Rightarrow p^{\prime}(x)=q^{\prime}(x)$. Finally, suppose $P$ is a unit or proper interval order with a representation in which $v \in V$ is assigned the interval $I_{v}=[L(v), R(v)]$. Define $L^{\prime}(x)$ and $R^{\prime}(x)$ as above. The prime representation has all endpoints distinct and it is straightforward to check that the unit or proper property is maintained. In a manner similar to above we can check that $R(x)<L(y) \Leftrightarrow R^{\prime}(x)<L^{\prime}(y)$ and hence the prime intervals also represent $P$.

Note that if two tolerances are equal the transformation in Lemma 7 maintains this. If in addition we want distinct tolerances, consider the following additional changes. Let $\delta$ be the smallest positive distance between two distinct points in $\left\{L^{\prime}(x), p^{\prime}(x), q^{\prime}(x), R^{\prime}(x) \mid x \in V\right\}$. Let $L^{\prime \prime}(x)=$ $L^{\prime}(x)-\delta / 10^{x}$ and $R^{\prime \prime}(x)=R^{\prime}(x)+\delta / 10^{x}$ and let $p^{\prime \prime}(x)=p^{\prime}(x)$ and $q^{\prime \prime}(x)=q^{\prime}(x)$. This preserves the distinct representation and yields distinct tolerances. However it does not preserve the unit property. For unit orders let $p^{\prime \prime \prime}(x)=p^{\prime}(x)-\delta / 10^{x}$ and $q^{\prime \prime \prime}(x)=q^{\prime}(x)+\delta / 10^{x}$ and let $L^{\prime \prime \prime}(x)=L^{\prime}(x)$ and $R^{\prime \prime \prime}(x)=R^{\prime}(x)$. This preserves the distinct representation and yields distinct tolerances. However it does not preserve the point-core property. All of the classes we have considered fit into at least one of the representations above except for unit point-core tolerance representations which by defintion have all tolerances equal. 


\section{The Hierarchy of Classes}

As discussed in the introduction, Figure 3 shows 18 classes of bounded bitolerance orders listed by their abbreviations from the table in Figure 2. For easy referral, the table in Figure 2 is repeated at the bottom of Figure 3. A few additional classes appear in Figure 3 as well. Point-core tolerance orders (3ai) are also known as 50\% tolerance orders since they have representations in which the tolerance is half the interval length. The terms "unit" and "proper" can be applied to interval orders in the natural way. A unit interval order is a poset that has an interval order representation in which each interval has the same length. Similarly, a proper interval order is a poset that has an interval order representation in which no interval properly contains another.

We first observe that the classes of bounded bitolerance orders in Figure 3 are ordered by inclusion so that a downward edge from class $S$ to class $T$ means that class $S$ contains class $T$. The restrictions in each category of the table in Figure 2 are listed from most restrictive to least restrictive. For example the "unit" restriction implies the "proper" restriction, thus we immediately get inclusions of the type (1ai) $\subseteq$ (2ai). Each of the inclusions shown in Figure 3 can be explained in this way and we know of no other inclusions.

The posets that appear along edges in Figure 3 will be shown to be separating examples. Their Hasse diagrams appear in Figure 4. Some of these examples also appear as separating examples in [3] where one separating example appears along each edge of the diagram in that paper. In this paper, besides including more classes, we also show the complete cut for all but one of the separating examples, that is, for each class in Figure 3, one can see exactly which of $\mathbf{2}+\mathbf{2}, \mathbf{3}+\mathbf{2}, \mathbf{3}+\mathbf{3}, \mathbf{4}+\mathbf{1}, A$, and $B$ is a member of that class. The edge between classes (1bi) and (2bi) is the only one for which we have no separating example.

In the remainder of this section, we justify the claims that are implied by Figure 3: (1) classes within a box are equivalent, and (2) any example that appears along an edge between two classes provides a separating example between those two classes. In section 3 we restrict attention to bipartite orders. In that setting, we prove that 16 of the 18 classes are equivalent, generalizing results from [3] and [6].

\subsection{Equivalent Classes}

The classes that appear together in a box in Figure 3 are equivalent classes. In Proposition 9 we show that the classes (1aii) and (2aii) are equivalent, and the classes (1bii) and (2bii) are equivalent. The former was observed by Fishburn and Reeds [5]. In Theorem 10 we prove the equivalence of the 


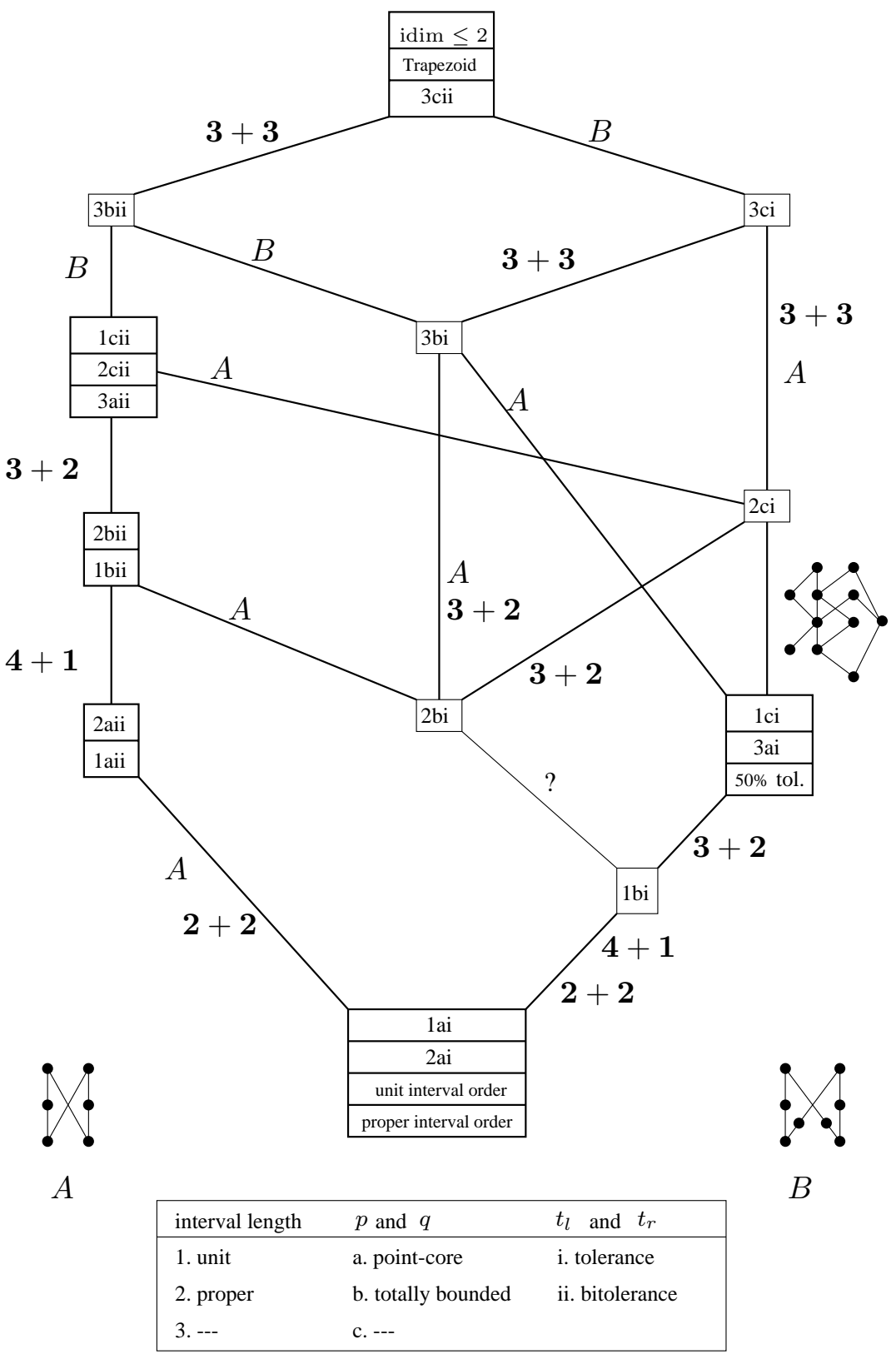

Figure 3: Some inclusions and separating examples between classes of bitolerance orders. 
classes in the bottom box in Figure 3. The following chart shows where the proofs of the other equivalences can be found.

\begin{tabular}{|ll|}
\hline Equivalence of classes ... & $\ldots$ proved in \\
\hline idim $\leq 2 /$ Trapezoid & {$[4]$} \\
idim $\leq 2 /(3$ cii $)$ & {$[11]$} \\
$(1$ cii $) /(2 \mathrm{cii}) /(3$ aii $)$ & {$[2]$ and $[11]$} \\
$(1 \mathrm{ci}) /(3 \mathrm{ai}) / 50 \%$ tolerance & {$[1]$ and $[11]$} \\
\hline
\end{tabular}

We begin by proving that bounded bitolerance orders can be represented so that $q(v) \leq p(v)$ for each element $v$ as discussed in the introduction. The transformation used in the next proposition has been used by other authors for different purposes. In [1] and [11] it is used to show that the class of unit tolerance orders is equivalent to the class of $50 \%$ tolerance orders, and in [9] the authors use this same transformation to show that tolerance graphs have representations in which all the intervals in the representation have a common intersection point. While this transformation is well-known, we believe that Propsition 8 has not appeared in the literature before.

Proposition 8. If $P=(V, \prec)$ is a bounded bitolerance order then $P$ has a representation $\langle\mathcal{I}, p, q\rangle$ in which $q(v) \leq p(v)$ for each $v \in V$.

Proof. Fix a bounded bitolerance representation $\langle\mathcal{I}, p, q\rangle$ of $P$ in which $I_{v}=$ $[L(v), R(v)]$. For any constant $M \geq 0$ the intervals $I_{v}^{\prime}=\left[L^{\prime}(v), R^{\prime}(v)\right]$ and tolerant points $p^{\prime}(v), q^{\prime}(v)$ defined by $L^{\prime}(v)=L(v)-M, q^{\prime}(v)=q(v)-M$, $p^{\prime}(v)=p(v)$ and $R^{\prime}(v)=R(v)$ give a bounded bitolerance representation of $P$. By choosing $M$ sufficiently large, we get a bounded bitolerance representation of $P$ in which $q^{\prime}(v) \leq p^{\prime}(v)$ for each $v \in V$.

Note that the transformation in the proof of Proposition 8 increases the length of every interval by $M$ and thus it preserves the properties of "unit" and "proper". In addition, it increases both the left and right tolerances by $M$, thus it also preserves the "tolerance" property.

There are several results in the literature comparing "unit" classes of interval and tolerance graphs to the analogous "proper" classes, for example, see $[1,2,12,10,14]$. The next proposition gives two additional results of this type. The latter is noted and used in [5], but we believe that no explicit proof of it has appeared previously.

Proposition 9. The classes of unit point-core bitolerance orders (1aii) and proper point-core bitolerance orders (2aii) are equivalent. The classes of unit totally bounded bitolerance orders (1bii) and proper totally bounded bitolerance orders (2bii) are equivalent.

Proof. The inclusions (1aii) $\subseteq$ (2aii) and (1bii) $\subseteq$ (2bii) are immediate, so we need only show the reverse inclusions. 
Observe that two bitolerance representations for which the relative order of the interval endpoints and tolerant points is the same represent the same order. Using this observation, we next show that a proper bitolerance representation can be transformed into a unit bitolerance representation of the same order. Afterwards we note that the transformation perserves the "point-core" and "totally bounded" properties.

We proceed by induction. Assume that any proper bitolerance representation $\langle\mathcal{I}, p, q\rangle$ of an order $P=(V, \prec)$ with $|V|<n$ can be transformed into a unit bitolerance representation of $P$. Furthermore, assume this can be accomplished so that the relative order of the set of endpoints and tolerant points is unchanged.

Let $P=(V, \prec)$ be a proper bitolerance order with $|V|=n$ and using Lemma 7 , fix a proper bitolerance representation $\langle\mathcal{I}, p, q\rangle$ of $P$ in which all endpoints and tolerant points are distinct. Let $x$ be the element with smallest left endpoint. Since the representation is proper, $R(x)$ is also the smallest right endpoint. By induction, fix a unit bitolerance representation $\left\langle\mathcal{I}^{\prime}, p^{\prime}, q^{\prime}\right\rangle$ of $P-x$ in which the points in $\left\{L^{\prime}(v), p^{\prime}(v), q^{\prime}(v), R^{\prime}(v)\right.$ : $v \in V-x\}$ appear in the same order as the corresponding points in $\{L(v), p(v), q(v), R(v): v \in V-x\}$.

For concreteness, translate and scale the new representation of $P$ $x$ so that the smallest left endpoint is $L^{\prime}(y)=0$ and $\left|I_{v}^{\prime}\right|=1$ for all $v$. Now place $R^{\prime}(x)$ so that its position with respect to the points in $\left\{L^{\prime}(v), p^{\prime}(v), q^{\prime}(v), R^{\prime}(v): v \in V-x\right\}$ matches the position of $R(x)$ with respect to the corresponding points in $\{L(v), p(v), q(v), R(v): v \in V-x\}$. We know $R^{\prime}(x)$ will be the smallest right endpoint in $\left\langle\mathcal{I}^{\prime}, p^{\prime}, q^{\prime}\right\rangle$, thus $R^{\prime}(x)<R^{\prime}(y)=1$. Set $L^{\prime}(x)=R^{\prime}(x)-1<0$, thus $L^{\prime}(x)$ will be the smallest left endpoint in $\left\langle\mathcal{I}^{\prime}, p^{\prime}, q^{\prime}\right\rangle$, as desired. Finally, place $p^{\prime}(x)$ (resp. $\left.q^{\prime}(x)\right)$ so that its position relative to points in $\left\{L^{\prime}(v), p^{\prime}(v), q^{\prime}(v), R^{\prime}(v): v \in V-x\right\}$ matches the position of $p(x)$ (resp. $q(x)$ ) with respect to the corresponding points in $\{L(v), p(v), q(v), R(v): v \in V-x\}$.

The new representation is unit. Furthermore, it has the same relative ordering of the interval endpoints and tolerant points as the original, so by our observation above, it represents the same order. If the original representation was point-core $(p(v)=q(v)$ for all $v)$ then since the ordering was maintained, $p^{\prime}(v)=q^{\prime}(v)$ for all $v$, and the new representation is pointcore. Likewise, if the original representation was totally bounded $(p(v) \leq$ $q(v)$ for all $v)$ then again since the ordering was maintained, $p^{\prime}(v) \leq q^{\prime}(v)$ for all $v$, and the new representation is totally bounded. This completes the proof.

We conclude this section with a proof that the classes in the bottom box of Figure 3 are equivalent.

Theorem 10. The following are equivalent statements about an order $P$. 
1. $P$ is a unit point-core tolerance order (1ai).

2. $P$ is a proper point-core tolerance order (2ai).

3. $P$ contains neither a $\mathbf{2 + 2}$ nor a $\mathbf{3}+\mathbf{1}$ as an induced suborder.

4. $P$ is a unit interval order.

5. $P$ is a proper interval order.

Proof. The implication (1) $\Longrightarrow(2)$ follows from the definitions of "unit" and "proper" restrictions. The equivalence of (3), (4) and (5) is implied by the work of [13], and written explicitly in terms of graphs in [12]. The proof also appears in [7] and elsewhere.

$(2) \Longrightarrow(3)$ Fix a proper point-core tolerance representation of $P=(V, \prec$ ) in which $v \in V$ is assigned the interval $I_{v}=[L(v), R(v)]$ and the splitting point $f(v)$. Since this is a tolerance representation, the splitting point $f(v)$ lies at the center of the interval $I_{v}$ for each $v \in V$, and as a reminder of this, we denote it by $c(v)$. For $x, y \in V$ we have $x \prec y$ if and only if $R(x)<c(y)$ and $c(x)<L(y)$. Hence two elements are comparable exactly when neither contains the other's center. In particular $x \| y$ if and only if $c(x) \in I_{y}$ or $c(y) \in I_{x}$. Note, also, in a proper tolerance representation the endpoints and center points of intervals occur in the same order, i.e. $R(x)<R(y)$ if and only if $L(x)<L(y)$ if and only if $c(x)<c(y)$.

Now, suppose $\mathrm{P}$ has an induced $\mathbf{2 + 2}$, that is a subset $x, y, z, w \in V$ such that $x \prec y$ and $z \prec w$ are the only comparabilities among them. Without loss of generality we may assume that $c(z) \leq c(x)$ and thus $R(z) \leq R(x)$. Now $x \prec y$ hence $c(x)<L(y)$ and so $c(z)<L(y)$. Also $x \prec y$ gives $R(x)<c(y)$ which implies $R(z)<c(y)$. These two inequalities together imply $z \prec y$, a contradiction.

Next suppose $\mathrm{P}$ has an induced $\mathbf{3 + 1}$, that is $x, y, z, w \in V$ such that $x \prec y \prec z$ are the only comparabilities among them. Since $x \prec y \prec z$ we have $R(x)<c(y)<L(z)$, also $R(y)<c(z)$ and $c(x)<L(y)$. Now $w \| x$ so either $c(w) \in I_{x}$ or $c(x) \in I_{w}$.

Case 1: If $c(w) \in I_{x}$ then $c(w) \leq R(x)<c(y)<L(z)$. Since $\mathrm{P}$ is proper $c(w)<c(y)$ implies $R(w)<R(y)$. This combined with $R(y)<c(z)$ gives $R(w)<c(z)$. And so we have $w \prec z$, a contradiction.

Case 2: If $c(x) \in I_{w}$ we have $L(w) \leq c(x)<L(y)$. Since $\mathrm{P}$ is proper this implies $R(w)<R(y)<c(z)$ and $c(w)<c(y)<L(z)$. Together these imply $w \prec z$, a contradiction.

This completes the proof of (2) implies (3).

(4) $\Longrightarrow$ (1) Let $\mathcal{I}=\left\{I_{v} \mid v \in V\right\}$ be a unit interval representation of $P=(V, \prec)$. By Lemma 7 we may assume the endpoints of intervals are distinct. Let $M=\left|I_{v}\right|$ for all $v \in V$, and let $\epsilon$ equal the smallest positive distance between any two distinct endpoints in the representation. It is not 
hard to see that the intervals $\left\{I_{v}=[L(v), R(v)] \mid v \in V\right\}$, and tolerant points $p(v)=L(v)+\epsilon / 2, q(v)=R(v)-\epsilon / 2$ for all $v \in V$, give a unit tolerance representation of $P$. Furthermore, the quantity $\operatorname{core}(v)=q(v)-p(v)=$ $M-\epsilon$ is constant for all $v \in V$ in this representation.

Now form a new set of intervals $\left\{I_{v}^{\prime} \mid v \in V\right\}$ by fixing $R(v)$ and $p(v)$ and shifting to the left $L(v)$ and $q(v)$ by core(v) for all $v \in V$. That is: $R^{\prime}(v)=R(v), p^{\prime}(v)=p(v)$, while $L^{\prime}(v)=L(v)-(M-\epsilon)$ and $q^{\prime}(v)=$ $q(v)-(q(v)-p(v))=p(v)$. Note that this is the same transformation used in the proof of Proposition 8. These new intervals give a unit point-core representation of an order $Q=\left(V, \prec^{\prime}\right)$ and we claim $Q=P$.

By shifting $L(v)$ and $q(v)$ by the same constant for all $v \in V$ they have the same relative order in $\mathrm{Q}$ as in $\mathrm{P}$; namely:

$$
q(x)<L(y) \Longleftrightarrow q(x)-(M-\epsilon)<L(y)-(M-\epsilon) \Longleftrightarrow q^{\prime}(x)<L^{\prime}(y) .
$$

Similarly, $R(v)$ and $p(v)$ are fixed for all $v \in V$ where

$$
R(x)<p(y) \Longleftrightarrow R^{\prime}(x)<p^{\prime}(y) .
$$

Thus $x \prec y$ in $P$ if and only if $x \prec " y$ in $Q$.

\subsection{Separating examples}

In this section we provide the details that justify the placement of orders $\mathbf{2}+\mathbf{2}, \mathbf{3}+\mathbf{2}, \mathbf{3}+\mathbf{3}, \mathbf{4}+\mathbf{1}, A$ and $B$ as separating examples in Figure 3. Whenever one of these orders appears along an edge in Figure 3 we prove it is a member of the larger class and not a member of the smaller class.

Several of these examples appear in [3]. The eleven element order shown was proven to separate the classes of unit tolerance orders (1ci) and proper tolerance orders (2ci) in [1]. The main result of [1], that the classes of unit tolerance orders and proper tolerance orders are unequal, provides a contrast to the many "unit = proper" results mentioned prior to Proposition 9. The edge between classes (1bi) and (2bi), for which we have no separating example, is another instance of comparing classes of unit and proper tolerance orders.

\subsubsection{The Orders $2+2$ and $3+3$}

Theorem 11. The orders $\mathbf{2}+\mathbf{2}$ and $\mathbf{3}+\mathbf{3}$ in Figure 4 separate the classes indicated in Figure 3.

Proof. It is easy to check that the order $\mathbf{2}+\mathbf{2}$ is a member of the classes (1aii/2aii) and (1bi), however it is not a member of (1ai/2ai) by Theorem 10. 


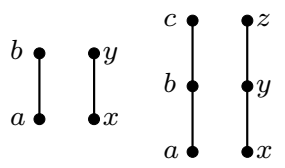

$2+2 \quad 3+3$

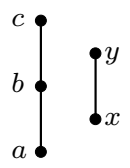

$3+2$

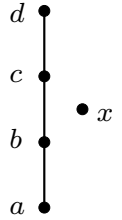

$4+1$

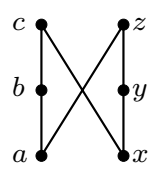

$A$

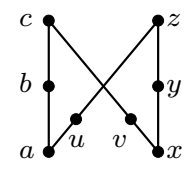

$B$

Figure 4: Orders that appear as separating examples in Figure 3.

We next show that whenever $\mathbf{3}+\mathbf{3}$ appears on an edge between two classes in Figure 3, it belongs to the larger class but not to the smaller class. First we show that $\mathbf{3}+\mathbf{3}$ belongs to the classes (3ci) and (3cii).

The following bounded tolerance representation of $\mathbf{3}+\mathbf{3}$ uses the labeling in Figure 4 and assigns $I_{v}=[L(v), R(v)], p(v)=R(v)$ and $q(v)=L(v)$ for all $v \in V: I_{a}=[1,10], I_{b}=[2,11], I_{c}=[3,12], I_{x}=[4,5], I_{y}=[6,7]$, $I_{z}=[8,9]$. By containment, it then follows that $\mathbf{3}+\mathbf{3}$ is a bounded bitolerance order (3cii).

In [3] it is shown that order $\mathbf{3}+\mathbf{3}$ is not a totally bounded bitolerance order (3bii). It then follows that $\mathbf{3}+\mathbf{3}$ is not a member of the more restrictive classes (3bi) and (1cii/2cii/3aii). The latter in turn implies that $\mathbf{3}+\mathbf{3}$ is not a member of the class $(2 \mathrm{ci})$.

\subsubsection{The Order $3+2$}

Lemma 12. The order $\mathbf{3}+\mathbf{2}$ is not a proper totally bounded bitolerance order (2bii).

Proof. Suppose we had a proper totally bounded bitolerance representation $\langle\mathcal{I}, p, q\rangle$ of the order $\mathbf{3}+\mathbf{2}$, labeled as in Figure 4 . First we show that the assumption $L(c) \leq q(x)$ leads to a contradiction. If $L(c) \leq q(x)$ then $L(c) \leq q(x)<L(y)$ since $x \prec y$. Using $a \prec b \prec c$ and the fact that the representation is totally bounded yields $R(a)<p(b) \leq q(b)<L(c)<L(y)$. However this means $I_{a}$ is completely to the left of $I_{y}$, contradicting $a \| y$.

Thus $q(x)<L(c)$. Now $x \| c$ so we must have $R(x) \geq p(c)$ and $b \prec c$ so $R(x) \geq p(c)>R(b)$. Since the representation is proper we conclude $L(x)>L(b)$.

Now $a \| y$ so either (i) $R(a) \geq p(y)$ or (ii) $L(y) \leq q(a)$. We show that each of these leads to a contradiction. If (i) holds then $R(a) \geq p(y)>R(x)$ since $x \prec y$. Using $L(x)>L(b)$ from above and $a \prec b$ we have $L(a) \leq$ $q(a)<L(b)<L(x)$. Together these imply $I_{x} \subseteq I_{a}$, contradicting the fact 
that the representation is proper. If (ii) holds then $L(y) \leq q(a)<L(b)<$ $L(x) \leq q(x)<L(y)$, a contradiction.

Theorem 13. The order $\mathbf{3}+\mathbf{2}$ separates the classes indicated in Figure 3.

Proof. The order $\mathbf{3}+\mathbf{2}$, labeled as in Figure 4, is a unit tolerance order (1ci) using the following representation: $I_{a}=[2,12], I_{b}=[3,13], I_{c}=[4,14]$, $I_{x}=[0,10], I_{y}=[6,16]$, and $t_{v}=\left|I_{v}\right|$ for $v=a, b, c$ and $t_{v}=1 / 2\left|I_{v}\right|$ for $v=x, y$. Therefore $\mathbf{3}+\mathbf{2}$ is also a member of the larger classes (2ci) and (1cii/2cii/3aii). In addition, the classes (1ci) and (3ai) are equal and (3ai) is contained in (3bi), thus $\mathbf{3}+\mathbf{2}$ is also a member of (3bi).

It remains to show that $\mathbf{3}+\mathbf{2}$ is not a member of the classes (1bii/2bii), (2bi), and (1bi). Since each of these classes is contained in (2bii), it suffices to show that $\mathbf{3}+\mathbf{2}$ is not a member of (2bii), which is done in Lemma 12 .

\subsubsection{The Order $4+1$}

Lemma 14. The order $\mathbf{4}+\mathbf{1}$ is not a proper point-core bitolerance order (1aii).

Proof. Suppose we had a proper point-core bitolerance representation $\langle\mathcal{I}, f\rangle$ of the order $\mathbf{4}+\mathbf{1}$, labeled as in Figure 4. Since $a \prec b \prec c \prec d$ we have the inequalities: $f(a)<L(b), R(a)<f(b)<L(c), R(b)<f(c)<L(d)$, $R(c)<f(d)$.

First consider the case in which $L(x)>f(a)$. Since $x \| a$ we must have $f(x)<R(a)$. Using the inequalities above and $L(c)<f(c)$, we obtain $f(x)<L(d)$. But $x \| d$ so we must have $R(x)>f(d)$. Now we have $R(x)>f(d)>R(c)$ and $L(x)<f(x)<R(a)<L(c)$, which implies that $I_{c}$ is a proper subset of $I_{x}$, a contradiction.

Therefore we must have $L(x) \leq f(a)$. Using the inequalities above, we have $L(x)<L(b)$, and since the representation is proper we must have $R(x)<R(b)$ which in turn implies $R(x)<L(d)<f(d)$. Since $x \| d$ we must have $L(d)<f(x)$ which implies $L(d)<f(x)<R(x)<L(d)$, a contradiction.

Theorem 15. The order $\mathbf{4}+\mathbf{1}$ separates the classes indicated in Figure 3.

Proof. The order $\mathbf{4}+\mathbf{1}$, labeled as in Figure 4, is a unit totally bounded tolerance order (1bi) using the following representation: $I_{a}=[0,20], I_{b}=$ $[11,31], I_{c}=[22,42], I_{d}=[33,53], I_{x}=[17,37]$, and $t_{x}=1$ and $t_{v}=10$ for $v=a, b, c, d$. Therefore $\mathbf{4}+\mathbf{1}$ is also a member of the larger class (1bii/2bii).

In Lemma 14 we show that $\mathbf{4}+\mathbf{1}$ is not a member of the class (1aii/2aii) and therefore it is not a member of the smaller class (1ai/2ai). 


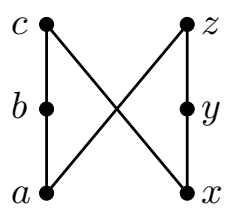

$A$

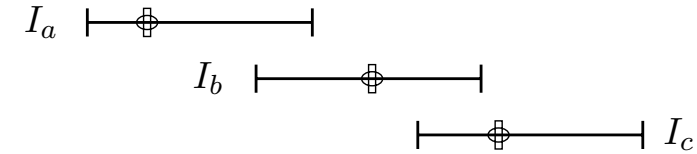

$I_{x} \longmapsto \oplus^{\prime}$

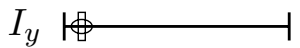

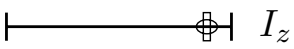

Figure 5: A unit point-core bitolerance representation of order $A$.

\subsubsection{The Order $A$}

Lemma 16. The order $A$ in Figure 4 is not a proper tolerance order (2ci).

Proof. Suppose $A=(V, \prec)$ were a proper tolerance order and fix a proper tolerance representation of $A$ in which $v \in V$ is assigned the interval $I_{v}=$ $[L(v), R(v)]$ and the tolerant points $p(v), q(v) \in I_{v}$. By symmetry we may assume $L(b) \leq L(y)$, and thus, since the representation is proper, $R(b) \leq$ $R(y)$.

First we show $t_{b}<t_{y}$. Since $y \prec z$ we have $R(y)<p(z)$ thus $R(b) \leq$ $R(y)<p(z)$. But $b \| z$ so we must have $L(z) \leq q(b)$. In addition, $q(y)<$ $L(z)$ (since $y \prec z$ ) so $q(y)<q(b)$. Hence $t_{b}=R(b)-q(b)<R(y)-q(y)=t_{y}$ as desired.

Next we show the opposite inequality $t_{y}<t_{b}$ must also hold, a contradiction. Since $a \prec b$ we have $q(a)<L(b)$. Combining this with our original assumption $L(b) \leq L(y)$ yields $q(a)<L(y)$. But $a \| y$ so $p(y) \leq R(a)$. Now $R(a)<p(b)$ (since $a \prec b$ ) so $p(y)<p(b)$. Using this last inequality and our assumption $L(b) \leq L(y)$ gives $t_{y}=p(y)-L(y)<p(b)-L(b)=t_{b}$, a contradiction.

Theorem 17. The order $A$ in Figure 4 separates the classes indicated in Figure 3.

Figure 5 shows a unit point-core bitolerance representation of order $A$ in which the oval and rectangle in interval $I_{i}$ mark the location of the splitting point $f(i)=p(i)=q(i)$. Thus $A$ is a member of the class (1aii), and therefore is also a member of the larger class (1bii/2bii).

The order $A$ is a totally bounded tolerance order (3bi) using the representation: $I_{a}=[1,11] p(a)=q(a)=6, I_{b}=[7,17] p(b)=q(b)=12$, $I_{c}=[13,23] \quad p(c)=q(c)=18, I_{x}=[7,9] \quad p(x)=q(x)=8, I_{y}=[9,15]$ $p(y)=10 q(y)=14$, and $I_{z}=[15,17] p(z)=q(z)=16$. Thus $A$ is also a member of the larger class $(3 \mathrm{ci})$. 
In Lemma 16 we proved that order $A$ is not a proper tolerance order (2ci). Hence is not a member of the smaller classes (2bi), and (2ai). This completes the proof.

\subsubsection{The Order $B$}

Lemma 18. The order B in Figure 4 is not a point-core bitolerance order (3aii).

Proof. For a contradiction, suppose $B$ were a point-core bitolerance order. Fix a point-core bitolerance representation of $B$ in which element $w$ is assigned interval $I_{w}=[L(w), R(w)]$ and splitting point $f(w)$. Recall that $i \prec j$ if and only if $R(i)<f(j)$ and $f(i)<L(j)$. Thus if $i \| j$ and one of these inequalities holds, then the other must be reversed.

By symmetry, we may assume that

$$
f(b) \leq f(y) .
$$

Claim 1: $f(z)<f(c)$.

Since $y \prec z$ we know $f(y)<L(z)$. Combining this with (1) yields $f(b)<L(z)$. But $b \| z$ so $f(z) \leq R(b)$. Now $b \prec c$ so $R(b)<f(c)$, which combined with the previous inequality yields $f(z)<f(c)$ as desired.

Claim 2: $f(x)<f(a)$.

Since $a \prec b$ we know $R(a)<f(b)$ which combined with (1) yields $R(a)<f(y)$. However, $a \| y$ so we must have $L(y) \leq f(a)$. This last inequality together with $f(x)<L(y)$ (because $x \prec y)$ gives $f(x)<f(a)$ as desired.

Now we consider the relative positions of $f(u)$ and $f(v)$. First suppose $f(u) \geq f(v)$. Since $x \prec v$ we have $R(x)<f(v)$ and thus

$$
R(x)<f(u) .
$$

But $a \prec u$ so $f(a)<L(u)$ and by Claim 2 we have $f(x)<L(u)$. This last inequality combined with (2) imply $x \prec u$, a contradiction.

Otherwise, $f(u)<f(v)$. Since $v \prec c$ we have $f(v)<L(c)$ which gives

$$
f(u)<L(c) .
$$

However, $u \prec z$ so $R(u)<f(z)$ and combining this with Claim 1 yields $R(u)<f(c)$. This last inequality together with (3) imply $u \prec c$, a contradiction.

Lemma 19. The order $B$ in Figure 4 is not a bounded tolerance order (3ci). 
Proof. For a contradiction, suppose $B=(V, \prec)$ were a bounded tolerance order. Fix a bounded tolerance representation of $B$ in which $w \in V$ is assigned the interval $I_{w}=[L(w), R(w)]$ and the tolerant points $p(w), q(w) \in$ $I_{w}$. Since this is a tolerance representation, we have $t_{w}=p(w)-L(w)=$ $R(w)-q(w)$ for each $w \in V$. By definition, $s \prec w$ if and only if $R(s)<p(w)$ and $q(s)<L(w)$. Thus if $s \| w$ and one of these two inequalities holds, then the other inequality must be reversed. We use this line of reasoning repeatedly.

Without loss of generality we may assume $q(a) \leq q(x)$.

Claim 1: $t_{y}<t_{b}$

Since $x \prec y$ we have $q(x)<L(y)$ so $q(a)<L(y)$. But $a \| y$ so we must have

$$
p(y) \leq R(a) .
$$

We also know $R(a)<p(b)$ because $a \prec b$. Combining this with (4) yields

$$
p(y)<p(b) .
$$

Now $R(x)<p(y)$ because $x \prec y$ so using (4) gives

$$
R(x)<R(a) .
$$

Combining (6) with $R(a)<p(b)$ above we get $R(x)<p(b)$. But $x \| b$ so we must have $L(b) \leq q(x)$. This together with $q(x)<L(y)$ (because $x \prec y$ ) yields

$$
L(b)<L(y) .
$$

Now using (5) and (7) we have, $t_{y}=p(y)-L(y)<p(b)-L(b)=t_{b}$ as desired. This proves claim 1.

Claim 2: $t_{v}<t_{u}$

The proof is the same as that of Claim 1 with $v$ in place of $y$ and $u$ in place of $b$.

We can obtain a bounded tolerance representation of the dual of the order $B$ by reflecting our representation about the $y$-axis. More precisely, the intervals $I_{w}^{\prime}=\left[L^{\prime}(w), R^{\prime}(w)\right]$ and tolerant points $p^{\prime}(w), q^{\prime}(w) \in I^{\prime}(w)$ form a bounded tolerance representation of the dual of $B$ where

$$
L^{\prime}(w)=-R(w), \quad R^{\prime}(w)=-L(w), \quad p^{\prime}(w)=-q(w), \text { and } q^{\prime}(w)=-p(w) .
$$

Note that all tolerances remain the same. Since $B$ is isomorphic to its dual, the proof of claim 1 can be applied again. Thus $q^{\prime}(c) \leq q^{\prime}(z) \Longrightarrow$ $t_{y}<t_{b}$ and $t_{u}<t_{v}$, the latter of which contradicts claim 2. Similarly, $q^{\prime}(z) \leq q^{\prime}(c) \Longrightarrow t_{b}<t_{y}$ and $t_{v}<t_{u}$, the former of which contradicts claim 1. In either case we get a contradiction. 


\begin{tabular}{|c|c|c|c|c|}
\hline $\mathrm{i}$ & $\mathrm{L}(\mathrm{i})$ & $\mathrm{p}(\mathrm{i})$ & $\mathrm{q}(\mathrm{i})$ & $\mathrm{R}(\mathrm{i})$ \\
\hline \hline $\mathrm{x}$ & 1 & 2 & 5 & 14 \\
$\mathrm{y}$ & 7 & 15 & 16 & 24 \\
$\mathrm{z}$ & 17 & 25 & 27 & 28 \\
$\mathrm{a}$ & 3 & 4 & 8 & 11 \\
$\mathrm{~b}$ & 9 & 12 & 19 & 21 \\
$\mathrm{c}$ & 20 & 22 & 26 & 27 \\
$\mathrm{u}$ & 10 & 13 & 16 & 23 \\
$\mathrm{v}$ & 6 & 15 & 18 & 21 \\
\hline
\end{tabular}

Figure 6: A totally bounded bitolerance representation of order $B$.

Theorem 20. The order B in Figure 4 separates the classes indicated in Figure 3.

Proof. Figure 6 gives a representation of the order $B$ as a totally bounded bitolerance order (3bii), thus $B$ is also a member of the larger class (3cii).

It remains to show that order $B$ is not a member of the classes (3ci), (3bi) and (1cii/2cii/3aii). In Lemma 18 we showed that the order $B$ is not a member of the class of point-core bitolerance orders (3aii/1dii/2dii), and in Lemma 19 we showed that the order $B$ is not in the class of bounded tolerance orders (3ci), thus $B$ is not a member of the more restrictive class (3bi). This accounts for all occurrences of $B$ in Figure 3 .

\section{The Bipartite Order Setting}

An ordered set $P=(V, \prec)$ is bipartite (also called height 2) if there are no three elements $x, y, z \in V$ with $x \prec y \prec z$. In Figure 3 , all the separating examples shown (except for the copies of $\mathbf{2}+\mathbf{2}$ at the bottom) are not bipartite orders. Indeed the following theorem shows that there are no bipartite separating examples because all of these classes (except the four in the bottom box) are equivalent in the bipartite domain. Figure 7 illustrates this result by showing the classes in Figure 3 in the case that only bipartite orders are considered.

Theorem 21 generalizes the results in [3] and [6], where fewer classes were considered. The proof of Theorem 21 involves the following condition from [3] on indexing the maximal and minimal non-isolates in a bipartite order.

A bipartite order $P$ satisfies the Indexing Condition if the minimal nonisolates can be indexed $\left\{x_{1}, x_{2}, \ldots, x_{m}\right\}$ and the maximal non-isolates can 


\begin{tabular}{|c|c|c|}
\hline & & 3ai \\
\hline 1aii & 2aii & 3aii \\
\hline $1 \mathrm{bi}$ & $2 \mathrm{bi}$ & $3 b i$ \\
\hline $1 \mathrm{bii}$ & $2 \mathrm{bii}$ & 3bii \\
\hline $1 \mathrm{ci}$ & $2 \mathrm{ci}$ & $3 \mathrm{ci}$ \\
\hline 1cii & 2cii & $3 \mathrm{cii}$ \\
\hline $\operatorname{idim} \leq 2$ & Trapezoid & $50 \%$ tol. \\
\hline & 1 ai & \\
\hline & 2ai & \\
\hline & \multicolumn{2}{|c|}{ init interval order } \\
\hline & \multicolumn{2}{|c|}{ roper interval order } \\
\hline
\end{tabular}

Figure 7: The hierarchy of Figure 3 collapsed in the case of bipartite orders.

be indexed $\left\{y_{1}, y_{2}, \ldots, y_{n}\right\}$ so that whenever $x_{i} \| y_{j}$ we have either $x_{k} \| y_{j}$ for all $k: 1 \leq k \leq i$, or $x_{i} \| y_{k}$ for all $k: 1 \leq k \leq j$.

Theorem 21. Within the domain of bipartite orders, all the classes in Figure 3 are equivalent, except for the four classes in the bottom box. Moreover, a bipartite order is a member of these equivalent classes if and only if it satisfies the indexing condition.

Proof. Because of the inclusions in Figure 3, it suffices to prove the following two results. (A) If $\operatorname{idim}(P) \leq 2$ then $P$ satisfies the indexing condition. (B) If $P$ satisfies the indexing condition, then $P$ is contained in each of the classes (1aii) and (1bi).

Result (A) is proven in [3]. In addition, the authors prove that if $P$ satisfies the indexing condition then $P$ is a unit tolerance order (1ci). Indeed, the construction in [3] produces a unit tolerance representation that is also totally bounded (1bi), however, the authors do not note this. Here we prove the remaining part of (B).

Let $P=(V, \prec)$ be a bipartite ordered set whose minimal non-isolated elements $X=\left\{x_{1}, x_{2}, \ldots, x_{m}\right\}$ and maximal non-isolated elements $Y=$ $\left\{y_{1}, y_{2}, \ldots, y_{n}\right\}$ are indexed according to the indexing condition. Let $Z$ be the set of isolated elements in $P$, thus $V=X \cup Y \cup Z$ is a partition of $V$. Finally, let $M=\max \{m, n\}$ and fix a constant $C \geq 4 M-1$.

We next construct a unit point-core bitolerance representation $\langle\mathcal{I}, f\rangle$ of 

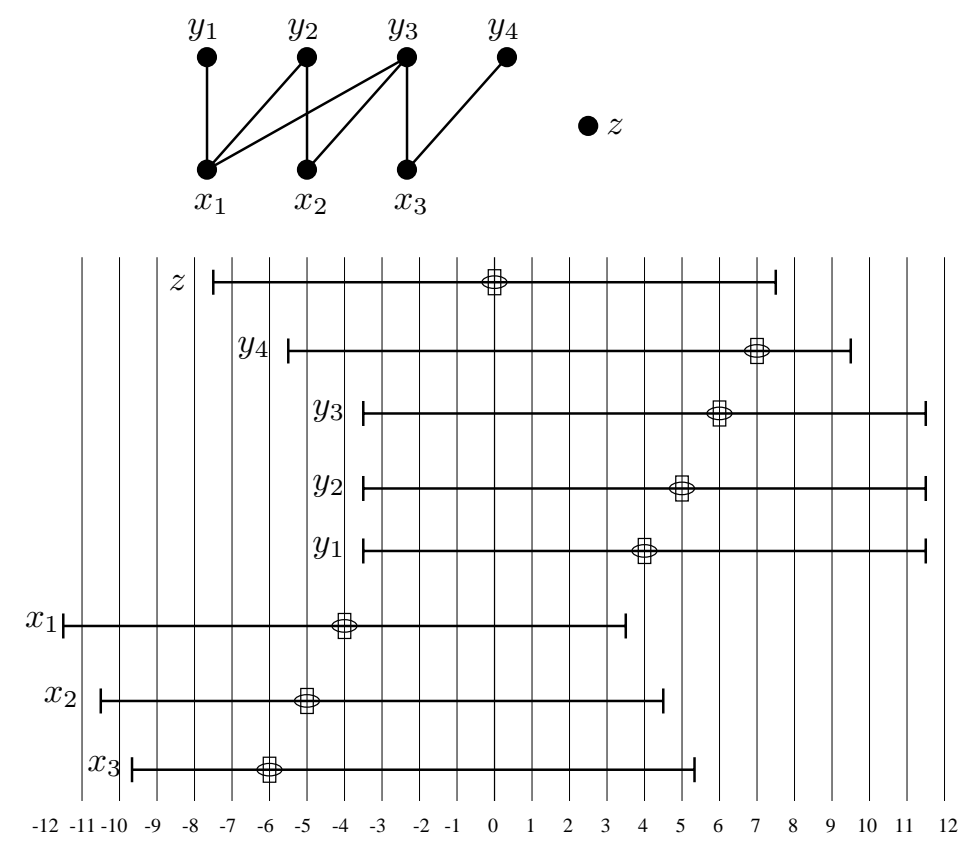

Figure 8: An ordered set and a unit point-core bitolerance representation of it.

$P$ in which each interval has length $C$. The construction is illustrated in Figure 8 in the case $C=4 M-1=15$.

- For each $x_{i} \in X$, let $f\left(x_{i}\right)=-(M+i-1)$.

- For each $y_{j} \in Y$, let $f\left(y_{j}\right)=M+j-1$.

- For each $x_{i} \in X$, let $L\left(x_{i}\right)=R\left(x_{i}\right)-C$ where

$$
R\left(x_{i}\right)= \begin{cases}f\left(y_{1}\right)-1 / 2 & \text { if } x_{i} \prec y_{1} \\ f\left(y_{k}\right)-1 / 2 & \text { if } x_{i} \prec y_{k} \text { and } x_{i} \| y_{l} \text { for all } l: 1 \leq l<k .\end{cases}
$$

- For each $y_{j} \in Y$, let $R\left(y_{j}\right)=L\left(y_{j}\right)+C$ where

$$
L\left(y_{j}\right)= \begin{cases}f\left(x_{1}\right)+1 / 2 & \text { if } x_{1} \prec y_{j} \\ f\left(x_{k}\right)+1 / 2 & \text { if } x_{k} \prec y_{j} \text { and } x_{l} \| y_{j} \text { for all } l: 1 \leq l<k .\end{cases}
$$

- For each isolate $z \in Z$, let $I_{z}=[-C / 2, C / 2]$ and $f(z)=0$. 
It remains to show that if $Q=\left(V, \prec^{\prime}\right)$ is the unit point-core bitolerance order with representation above then $Q=P$. We show $v \prec w$ if and only if $v \prec^{\prime} w$ for all $v, w \in V$ using four cases.

Case 1: $v, w \in X$ :

Let $v=x_{i}$ and $w=x_{j}$. Since every pair of elements in $X$ is incomparable in $P$, we must show $x_{i} \| x_{j}$ in $Q$. Note that

$L\left(x_{j}\right)=R\left(x_{j}\right)-C<(2 M-1)-(4 M-1)=-2 M<-(M+m-1) \leq q\left(x_{i}\right)$,

so $x_{i} \nprec^{\prime} x_{j}$. By symmetry, $x_{j} \nprec^{\prime} x_{i}$, thus $x_{i} \| x_{j}$ in $Q$.

Case 2: $v, w \in Y$ :

The proof is similar to Case 1 .

Case 3: $v \in V, w=z \in Z$ :

Since $z$ is an isolated element of $P$, we know $z \| v$ in $P$ and must show $z \| v$ in $Q$. We know $v \nprec^{\prime} z$ because $R(v)>0=f(z)$ and $z \nprec^{\prime} v$ because $f(z)=0>L(v)$. Hence $z \| v$ in $Q$.

Case 4: $v=x_{i} \in X, w=y_{j} \in Y$ :

If $x_{i} \prec y_{j}$ then by the definitions of $R\left(x_{i}\right)$ and $L\left(y_{j}\right)$, we know $R\left(x_{i}\right)<$ $f\left(y_{j}\right)$ and $L\left(y_{j}\right)>f\left(x_{i}\right)$, so $x_{i} \prec^{\prime} y_{j}$.

Otherwise, $x_{i} \| y_{j}$ in $P$. By the indexing condition, either (i) $x_{i} \| y_{l}$ in $P$ for all $l: 1 \leq l \leq j$, or (ii) $x_{l} \| y_{j}$ in $P$ for all $l: 1 \leq l \leq i$. In case (i), $R\left(x_{i}\right)>f\left(y_{j}\right)$ by definition of $R\left(x_{i}\right)$, and in case (ii), $L\left(y_{j}\right)<f\left(x_{i}\right)$ by definition of $L\left(y_{j}\right)$. In either case, $x_{i} \nprec^{\prime} y_{j}$. We know $y_{j} \nprec^{\prime} x_{i}$ since

$$
R\left(y_{j}\right)=L\left(y_{j}\right)+C>(-2 M+1)+(4 M-1)=2 M>R\left(x_{i}\right) \geq f\left(x_{i}\right) .
$$

Thus $x_{i} \| y_{j}$ in $Q$ as desired. This completes the proof.

\section{Acknowledgement}

The authors would like to thank Martin Golumbic for helpful conversations which improved this paper.

\section{References}

[1] K. Bogart, P. Fishburn, G. Isaak, and L. Langley. Proper and unit tolerance graphs. Discrete Applied Math., 60:37-51, 1995.

[2] K.P. Bogart and G. Isaak. Proper and unit bitolerance orders and graphs. Discrete Math., 181:37-51, 1998.

[3] K.P. Bogart and A.N. Trenk. Bipartite tolerance orders. Discrete Mathematics, 132:11-22, 1994. Corrigendum to "Bipartite Tolerance Orders" Discrete Math. 145 (1995) 347. 
[4] I. Dagan, M.C. Golumbic, and R.Y. Pinter. Trapezoid graphs and their coloring. Disrete Applied Math., 21:35-46, 1988.

[5] P.C. Fishburn and J.A. Reeds. Counting split semiorders. To appear in ORDER.

[6] P.C. Fishburn and W.T. Trotter. Split semiorders. Discrete Math., 195:111-126, 1999.

[7] M.C. Golumbic. Algorithmic Graph Theory and Perfect Graphs. Academic Press, San Diego, CA, 1980.

[8] M.C. Golumbic and C.L. Monma. A generalization of interval graphs with tolerances. Congressus Numerantium, 35:321-331, 1982.

[9] M.C. Golumbic, C.L. Monma, and W.T. Trotter. Tolerance graphs. Discrete Applied Math., 9:157-170, 1984.

[10] M.S. Jacobson and F.R. McMorris. Sum-tolerance proper interval graphs are precisely sum-tolerance unit interval graphs. J. Combinatorics, Infomation and System Sciences, 16:25-28, 1991.

[11] L. Langley. Interval tolerance orders and dimension. PhD thesis, Dartmouth College, June 1993.

[12] F.S. Roberts. Indifference graphs. In F. Harary, editor, Proof Techniques in Graph Theory, pages 139-146. Academic Press, New York, 1969.

[13] D. Scott and P. Suppes. Foundational aspects of theory of measurement. J. of Symbolic Logic, 23:113-128, 1958.

[14] R. Shull and A.N. Trenk. Unit and proper bitolerance digraphs. J. of Graph Theory, 24:193-199, 1997. 